\section{Prise En Charge Des Plaies Pénétrantes De L'abdomen À L'hôpital National De Niamey}

\section{ESJ Natural/Life/Medical Sciences}

\section{Hama Y,}

Département de chirurgie de la faculté des sciences de la santé de l’Université Abdou Moumouni de Niamey-Niger, Département de chirurgie de l'hôpital national de Niamey-Niger

\section{Mohamed A.A,}

Département de chirurgie de la faculté des sciences de la santé de l’Université Abdou Moumouni de Niamey-Niger, Département de chirurgie de l'hôpital national de Niamey-Niger

\section{Kadi I,}

Département de chirurgie de l'hôpital national de Niamey-Niger

\section{James Didier L,}

Département de chirurgie de la faculté des sciences de la santé de l’Université Abdou Moumouni de Niamey-Niger, Département de chirurgie de l'hôpital national de Niamey-Niger

\section{Sani R,}

Département de chirurgie de la faculté des sciences de la santé de l'Université Abdou Moumouni de Niamey-Niger, Département de chirurgie de l'hôpital national de Niamey-Niger

Submitted: 08 January 2020

Accepted: 03 September 2020

Published: 31 December 2020

Corresponding author:

Hama Younssa

DOI: $10.19044 /$ esj.2020.v16n36p212

(c) Copyright 2020 Hama Y,

Distributed under Creative Commons BY-NC-ND 4.0 OPEN ACCES

\section{Cite as:}

Hama Y, Mohamed AA, Kadi I., James Didier L, Sani R . (2020). Prise en charge des plaies pénétrantes de l'abdomen à l'hôpital national de Niamey. European Scientific Journal, ESJ, 16 (36), 1. https://doi.org/10.19044/esj.2020.v16n36p212

\section{Résumé}

Objectif : rapporter les résultats de la prise en charge des plaies pénétrantes de l'abdomen à l'hôpital national de Niamey au Niger. Patients et méthodes : il s'agissait d'une étude rétrospective et descriptive sur une période de 2 ans. L'étude a concerné tous les patients de tout âge pris en charge à l'hôpital national de Niamey pour plaie pénétrantes de l'abdomen. Résultats : l'étude incluait 51 patients, soit $0,88 \%$ des traumatismes. Il s'agissait de 6 femmes et 45 hommes, soit un sex ratio de 7,5. L'âge moyen des patients était de 28 ans $[ \pm 10,2]$. Le délai moyen d'admission était de 4,86 heures $[ \pm 2,08]$. Dans $84,32 \%$ des cas les patients étaient transportés à l'hôpital dans un véhicule non médicalisé. Les agressions représentaient 78,44\% des circonstances de survenue et la plaie abdominale s'intégrait dans un contexte de polytraumatisme chez $21,53 \%$ des patients. Une éviscération était présente chez $31,37 \%$ des patients. Le traitement était opératoire chez 90,19\% des patients avec $19,56 \%$ de laparotomie non thérapeutique. L'organe le plus lésé était l'intestin grêle dans 31,37\% des cas suivi du colon dans $23,52 \%$ des cas. La morbidité globale était de 33,37\% dominée par les hémorragies postopératoires dans $8,69 \%$ des cas. La mortalité était de $13,73 \%$ avec 50,14\% de décès en postopératoire immédiat par hémorragie secondaire ; 28,58\% de décès par retard de l'hémostase chirurgicale et $14,28 \%$ de décès par défaut de compensation des pertes sanguines en urgence.Conclusion : les plaies de l'abdomen sont fréquentes chez l'adulte jeune de sexe masculin. Elles sont souvent dues à une agression par armes blanches avec un taux élevé de décès. 
European Scientific Journal, ESJ

December 2020 edition Vol.16, No.36
ISSN: 1857-7881 (Print) e - ISSN 1857-7431

\section{Subject: Medicine}

Mots-clés: Plaies Pénétrantes De L'abdomen, Plaies Par Armes Blanches, Éviscération, Plaie Abdominale. 


\section{Management Of Penetrating Abdominal Wounds}

\section{Hama Y,}

Département de chirurgie de la faculté des sciences de la santé de l’Université Abdou Moumouni de Niamey-Niger, Département de chirurgie de l'hôpital national de Niamey-Niger

\section{Mohamed A.A,}

Département de chirurgie de la faculté des sciences de la santé de l’Université Abdou Moumouni de Niamey-Niger, Département de chirurgie de l'hôpital national de Niamey-Niger

\section{Kadi I,}

Département de chirurgie de l'hôpital national de Niamey-Niger

\section{James Didier $\mathbf{L}$,}

Département de chirurgie de la faculté des sciences de la santé de l’Université Abdou Moumouni de Niamey-Niger, Département de chirurgie de l'hôpital national de Niamey-Niger

\section{Sani $R$,}

Département de chirurgie de la faculté des sciences de la santé de l’Université Abdou Moumouni de Niamey-Niger, Département de chirurgie de l'hôpital national de Niamey-Niger

\section{Abstract}

Objective: to report the results of the management of the penetrating abdominal wounds at the national hospital of Niamey.

Patients and method: it was a retrospective and descriptive study over 2 years. The study included the patients of all ages managed for penetrating abdominal wounds at national hospital of Niamey in Niger.

Results: the study includes 51 patients whether $0.88 \%$ of trauma. They were 45 men and 6 women, with a sex ratio of 7.5. The average age was 28 [ \pm 10.2$]$ years old. The average intake time was 4.86 [2.08] hours. In 84 . $32 \%$ of cases, patients were transported to the hospital in non-medical vehicle. The assault accounted for $78.44 \%$ of the circumstances of the occurrence and the penetrating abdominal wound was integrated into a polytrauma in $21.53 \%$ of cases. An evisceration was present in $31.37 \%$ of cases. The management was operative in $90.19 \%$ of cases with $19.56 \%$ of non-therapeutic laparotomy. The most injured organ was the small intestine in $31.37 \%$ of cases followed by the colon in $23.52 \%$ of cases. The overall morbidity was $33.37 \%$, 
dominated by postoperative hemorrhages in $8.69 \%$ of cases. The mortality was $13.73 \%$ with $50.14 \%$ of immediate deaths by secondary hemorrhage, $28.58 \%$ of deaths by delay of the surgical hemostasis and $14.28 \%$ of deaths by a failure of compensation of the blood losses in emergency room.

Conclusion: the penetrating abdominal wounds are common in young adult males. They are often due to a stabbing assault with a high death rate.

Keywords: Penetrating Abdominal Wounds, Stabbing Wound, Evisceration, Abdominal Wound

\section{Introduction}

Les plaies pénétrantes de l'abdomen réalisent une rupture de la continuité de la paroi abdominale avec effraction du péritoine pariétale. Les plaies pénétrantes de l'abdomen sont souvent caractérisées par leur gravité immédiate par atteinte d'un gros vaisseau ou par dilacération d'un organe plein et secondairement par leur risque septique. La fréquence et la gravité des plaies de l'abdomen sont variable (Störmann, 2016). Toute fois la fréquence des plaies de l'abdomen est en augmentation en Afrique (Fanomezantsoa, 2015) du fait de l'occidentalisation des villes africaines (Coquery-Vidrovitch, 2006), mais aussi de l'augmentation des zones de conflit dans cette partie du monde (Vincent, 2013). La prise en charge des plaies de l'abdomen, en dépit des recommandations (Biffl, 2015) n’est pas toujours aisée du fait de la possibilité d'atteinte simultanée d'organes relevant de spécialités chirurgicales différentes mais aussi de la possibilité d'atteinte simultanée des compartiments péritonéal, rétropéritonéal et thoracique (Barbois, 2016). Cette prise en charge pluridisciplinaire, qui se fait au mieux à travers des "'trauma system"' (MacKenzie, 2006), a lieu le plus souvent en Afrique dans des hôpitaux généraux à l'instar de l'hôpital national de Niamey. Cette étude a pour objectif de rapporter les résultats de la prise en charge des plaies abdominales en service de chirurgie générale à l'hôpital national de Niamey.

\section{Patients et méthode}

Il s'agissait d'une étude rétrospective descriptive et analytique menée d'octobre 2011 à octobre 2013. L'étude était réalisée dans les services de chirurgie générale A, B et C2 de l'hôpital national de Niamey (Niger). L'étude a concerné les patients des 2 sexes pris en charge pour plaie pénétrante de l'abdomen à l'hôpital national de Niamey durant la période de l'étude. Les données ont été colligées à partir des dossiers des patients et des registres du service des urgences, des registres de comptes rendus opératoires et des registres des services de chirurgie générale A, B et C2. Les variables étudiées étaient l'âge, le sexe, le délai d'admission, les circonstances du traumatisme, l'instabilité hémodynamique définie par une tension artérielle systolique 
inférieure ou égale à $90 \mathrm{~mm} \mathrm{Hg}$ et/ou une fréquence cardiaque supérieure ou égale à 120 pulsations par minute, les lésions abdominales, les lésions associées, le mode de traitement, le geste chirurgical réalisé ou une laparotomie non thérapeutique définie par la découverte de lésions ne nécessitant aucun geste thérapeutique en cas de traitement opératoire, la morbidité et la mortalité des plaies pénétrantes de l’abdomen.

\section{Résultats}

La série comportait 51 cas de plaies pénétrantes de l'abdomen représentant $35,17 \%$ des traumatismes abdominaux et $0,88 \%$ de l'ensemble des urgences chirurgicales; soit en moyenne 25,5 plaies pénétrantes de l'abdomen par an et 2 plaies pénétrantes de l'abdomen par mois avec un pic de fréquence de 4 à 5 plaies pénétrantes de l'abdomen en octobre et en novembre. Il s'agissait de 6 femmes et de 45 hommes, soit un sexe ratio de 7,5 . L'âge moyen des patients était de $28[ \pm 10,2]$ ans et $58,82 \%$ des patients avaient moins de 30 ans. Le transport des patients du lieu de la blessure à l'hôpital était médicalisé pour 15,68\% des patients, 84,32 \% des patients étaient amenés à l'hôpital par des proches. Le délai moyen d'admission des patients aux urgences était de 4,86 [ $\pm 2,08]$ heures avec des extrêmes de 30 minutes à 48 heures ; cependant 60,78\% des patients étaient admis au-delà de la première heure après la blessure. Les circonstances de survenue de la blessure sont résumées dans le tableau I. A l'admission 37,25\% des patients avaient une instabilité hémodynamique $(\mathrm{n}=19)$. Cette instabilité hémodynamique était 2 fois plus fréquente en cas de plaie par arme à feu.

Tableau I : répartition des patients en fonction des circonstances de survenue de la plaie abdominale

\begin{tabular}{|c|c|c|}
\hline & Nombre & Pourcentage \\
\hline Agression par arme blanche & 31 & 60,79 \\
\hline Agression par arme à feu & 9 & 17,65 \\
\hline Accident de travail & 4 & 7,84 \\
\hline Tentative d'autolyse & 3 & 5,88 \\
\hline Accident domestique & 3 & 5,88 \\
\hline Accident de la voie publique & 1 & 1,96 \\
\hline TOTAL & 51 & 100 \\
\hline
\end{tabular}

Une échographie abdominale et un scanner abdominal avaient été réalisés chez 11,70\% des patients (chez les 5 patients non opérés et chez un patient opéré). Le traitement était non opératoire chez 9,81\% des patients et opératoire chez 90,19\% patients; cependant la laparotomie était non thérapeutique chez $19,56 \%$ des opérés $(n=9)$. Les indications opératoires étaient l'éviscération chez $31,37 \%$ des patients (photo 1), l'instabilité hémodynamique persistante chez 19,60\% des patients, la laparotomie exploratrice chez $19,60 \%$ des patients, la péritonite chez 15,68\% des patients, l'issue de liquide digestif par la plaie chez $3,92 \%$ des patients, et plaie 
thoraco-abdominale ou abdomino-pelvienne chez 9,83\% des patients. L'organe extériorisé était l'intestin grêle chez 23,52 \% des patients, suivi de l'épiploon chez $11,76 \%$ des patients, du colon chez 5,88\% des patients et de l'estomac chez 3,92\% des patients. Les indications opératoires ayant conduit à une laparotomie non thérapeutique étaient l'éviscération chez 6 patients (extériorisation de l'épiploon chez 4 patients et extériorisation de l'intestin grêle chez 2 patients) et la péritonite chez 2 patients. La plaie abdominale était unique chez $78,43 \%$ des patients et multiple chez $21,57 \%$ des patients. La plaie était thoraco-abdominale chez 3,92\% des patients et abdomino-périnéale chez un patient et chez $21,29 \%$ des patients elle intéressait en plus le compartiment rétropéritonéal (photo 2).

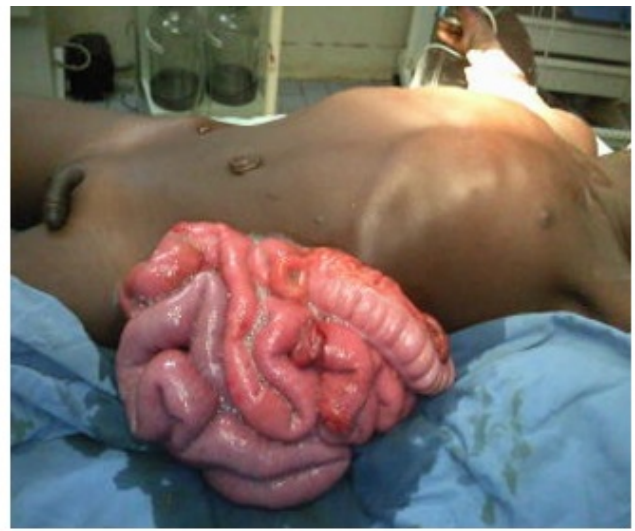

Figure 1 : éviscération traumatique par accident de la voie publique avec section de l'intestin grêle (flèches)

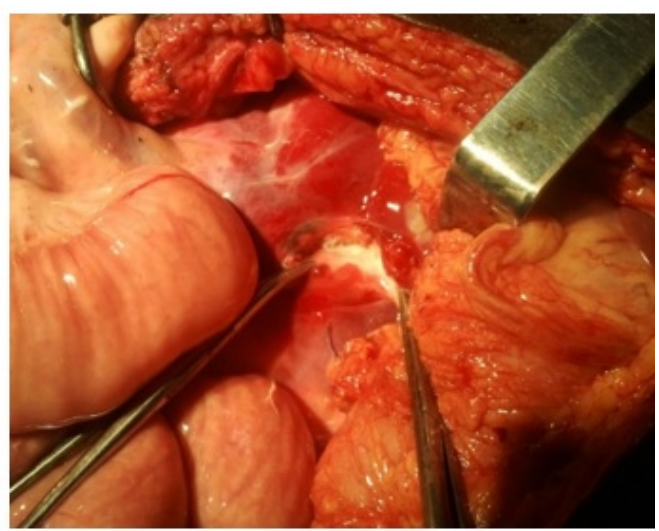

Photo 2 : plaie rétropéritonéale avec section du canal thoracique secondaire à une agression par arme blanche avec issue de lymphe

Les lésions du contenu abdominal (photo3) et les lésions associées sont résumées dans les tableaux II et III. Chaque patient avait en moyenne 1,95 organe intra-abdominal lésé et au moins une lésion extra-abdominale associée. Cette association lésionnelle réalisait un polytraumatisme chez $21,57 \%$ des patients. Cependant les armes à feu (avec en moyenne 3 organes lésés par patient) avaient occasionné plus de lésions d'organes que les autres circonstances de survenue de la plaie pénétrante de l'abdomen. Les gestes thérapeutiques réalisés pour les lésions du contenu abdominal et les lésions associées sont résumés dans les tableaux IV et V.

Tableau II : répartition des patients selon les lésions du contenu abdominal

\begin{tabular}{|c|c|c|}
\hline & Nombre & Pourcentage \\
\hline Estomac & 8 & 15,68 \\
\hline Jéjuno-iléon & 16 & 31,37 \\
\hline Duodénum & 2 & 3,92 \\
\hline Colon & 12 & 23,52 \\
\hline Rectum & 1 & 1,96 \\
\hline
\end{tabular}




\begin{tabular}{|c|c|c|}
\hline Foie & 4 & 7,84 \\
\hline Vessie & 2 & 3,92 \\
\hline Rate & 2 & 3,92 \\
\hline Reins & 1 & 1,96 \\
\hline Pancréas & 2 & 3,92 \\
\hline Grand Epiploon & 11 & 21,56 \\
\hline Mésentère & 9 & 17,65 \\
\hline Mésocolon & 4 & 7,84 \\
\hline $\begin{array}{c}\text { Veine mésentérique supérieure } \\
\text { (VMS) }\end{array}$ & 1 & 1,96 \\
\hline Canal thoracique & 1 & 1,96 \\
\hline
\end{tabular}

Tableau III : répartition des patients en fonction des lésions associées

\begin{tabular}{|c|c|c|}
\hline & Nombre & Pourcentage \\
\hline Plaie du diaphragme & 2 & 3,92 \\
\hline Fracture de côte & 2 & 3,92 \\
\hline Plaie du poumon & 1 & 1,96 \\
\hline Hémothorax et hémopneumothorax & 4 & 7,84 \\
\hline Fracture de membre & 7 & 13,72 \\
\hline Luxation de la hanche & 1 & 1,96 \\
\hline Fracture du bassin & 1 & 1,96 \\
\hline Plaies des muscles squelettiques & 3 & 5,88 \\
\hline Plaies cutanée extra-abdominales & 30 & 58,82 \\
\hline
\end{tabular}

Tableau IV : gestes thérapeutiques réalisés sur les lésions abdominales

\begin{tabular}{|c|c|c|c|c|c|c|}
\hline & Suture & $\begin{array}{c}\text { Résection } \\
+/- \\
\text { anastomose }\end{array}$ & $\begin{array}{l}\text { Résection } \\
+ \text { stomie }\end{array}$ & $\begin{array}{c}\text { Packing } \\
\text { et/ou } \\
\text { hémostase }\end{array}$ & Abstention & Total \\
\hline Estomac & $\begin{array}{c}8 \\
(17,39) \\
\end{array}$ & - & - & - & - & $8(17,39)$ \\
\hline $\begin{array}{c}\text { Jéjuno- } \\
\text { iléon }\end{array}$ & $8(17,39)$ & $6(13,04)$ & $2(4,34)$ & - & - & $16(34,78)$ \\
\hline Duodénum & $2(4,34)$ & - & - & - & - & $2(4,34)$ \\
\hline Colon & $8(17,39)$ & $2(4,34)$ & $2(4,34)$ & - & - & $12(26,06)$ \\
\hline Rectum & $1(2,17)$ & - & - & - & - & $1(2,17)$ \\
\hline Foie & - & $1(2,17)$ & - & $1(2,17)$ & $2(4,34)$ & $4(8,70)$ \\
\hline Vessie & $2(4,34)$ & - & - & - & - & $2(4,34)$ \\
\hline Rate & - & $2(4,34)$ & - & - & - & $2(4,34)$ \\
\hline Rein & - & - & - & - & $1(2,17)$ & $1(2,17)$ \\
\hline Pancréas & $2(4,34)$ & - & - & - & - & $2(4,34)$ \\
\hline Epiploon & - & $9(19,56)$ & - & - & - & $9(19,56)$ \\
\hline Mésentère & $5(10,86)$ & - & - & - & $4(8,70)$ & $9(19,56)$ \\
\hline Mésocolon & $2(4$ ?34) & - & - & - & $2(4,34)$ & $4(8,70)$ \\
\hline VMS & $1(2,17)$ & - & - & - & - & $1(2,17)$ \\
\hline $\begin{array}{c}\text { Canal } \\
\text { thoracique }\end{array}$ & - & - & - & $\begin{array}{c}\text { 1(fermeture } \\
\text { du } \\
\text { péritoine }\end{array}$ & - & $1(2,17)$ \\
\hline
\end{tabular}




\begin{tabular}{|l|l|l|l|c|l|l|}
\hline & & & & $\begin{array}{c}\text { pariétal } \\
\text { postérieur })\end{array}$ & & \\
\hline
\end{tabular}

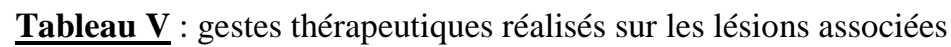

\begin{tabular}{|c|c|c|}
\hline & Geste réalisé & $\mathbf{n ~ ( \% )}$ \\
\hline Plaie du diaphragme & $\begin{array}{c}\text { Suture du diaphragme avec drainage } \\
\text { thoracique }\end{array}$ & $2(4,34)$ \\
\hline Fracture de côte & Traitement non opératoire & $2(4,34)$ \\
\hline Plaie du poumon & $\begin{array}{c}\text { Suture du poumon avec drainage } \\
\text { thoracique }\end{array}$ & $1(2,17)$ \\
\hline $\begin{array}{c}\text { Hémothorax et } \\
\text { hémopneumothorax }\end{array}$ & $\begin{array}{c}\text { Drainage thoracique } \\
\text { Fracture de membre }\end{array}$ & $4(8,70)$ \\
\hline Luxation de la hanche & Réduction orthopédique & $7(15,21)$ \\
\hline Fracture du bassin & Exofixation du bassin & $1(2,17)$ \\
\hline Plaies des muscles squelettiques & Parage et suture & $3(6,52)$ \\
\hline $\begin{array}{c}\text { Plaies cutanée extra- } \\
\text { abdominales }\end{array}$ & Parage et suture & $30(65,21)$ \\
\hline
\end{tabular}

Le séjour hospitalier moyen était de 9,25 [ $\pm 3,50]$ jours. Les complications immédiates étaient dominées par l'hémorragie secondaire survenue chez 8,69\% des opérés $(n=4)$, la suppuration pariétale survenue chez 4,34\% des opérés $(n=2)$, la péritonite postopératoire par lâchage de suture, une éviscération postopératoire et un retard de reprise du transit au-delà de 72 heures survenue chacun chez un patient. La mortalité globale était de 13,73\% $(\mathrm{n}=7)$. Cette mortalité était 1,5 fois plus fréquente en cas de plaie abdominale par arme à feu (22,2\% VS 14,2\%). Deux patients (28,58\% des décédés) étaient décédés avant l'intervention chirurgicale par hémorragie. Un patient (14,28\% des décédés) était décédé au moment de l’induction anesthésique et les autres patients (57,14\% des décédés) étaient décédés en postopératoire immédiat dans un tableau de choc hypovolémique.

\section{Discussion}

Les plaies de l'abdomen sont relativement moins fréquentes que les contusions de l'abdomen à l'hôpital national de Niamey. Elles représentaient 47\% des traumatismes abdominaux en 2000 (Sani, 2004) et 35,17\% de ces traumatismes dans notre série. Mais elles sont en augmentation, en effet de 21 cas annuels entre 1985 et 1999 (Naomie, 2002), elles sont passées à 25,5 cas annuels dans notre série avec un pic de fréquence en octobre et en novembre. Ce pic de fréquence correspond à la période de retour des populations rurales en ville (exode rurale) augmentant probablement le banditisme urbain. Les plaies pénétrantes de l'abdomen, à Niamey comme partout ailleurs (Störmann, 2016), (MacKenzie, 2006), (Ayité, 1996) surviennent surtout chez l’adulte 
jeune de sexe masculin ; ainsi l'âge moyen des patients de notre série était de $28[ \pm 10,2]$ ans avec $58,82 \%$ des patients qui étaient âgés de moins de 30 ans. Ces plaies pénétrantes de l'abdomen étaient dues pour 60,79\% aux agressions par arme blanche et pour 17,65\% aux agressions par arme à feu dans notre série soit 78,44\%.

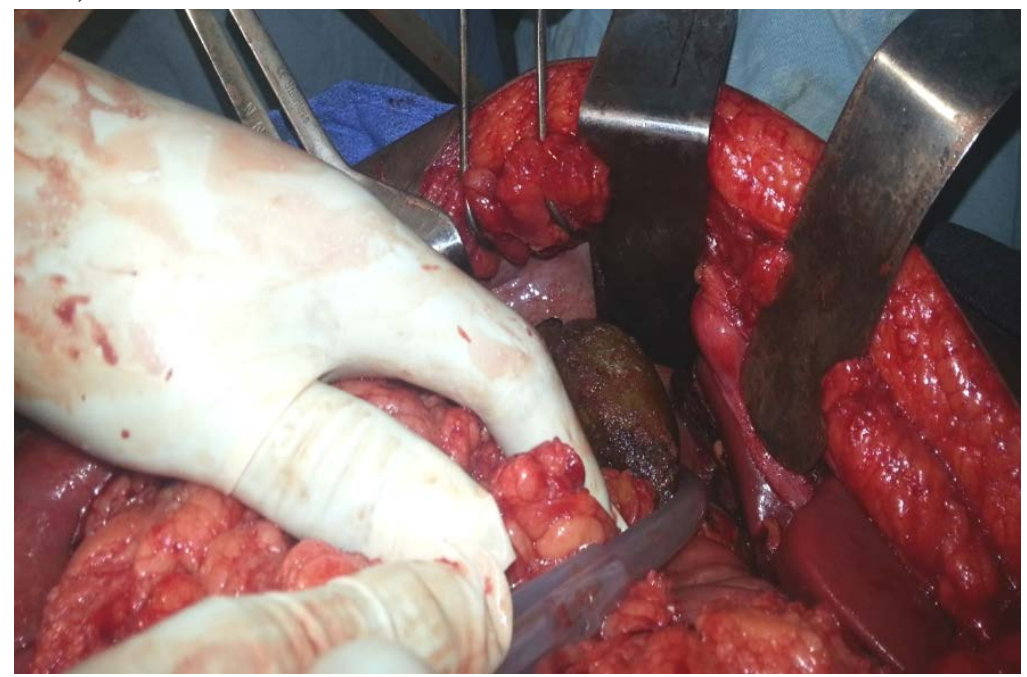

Photo3. Nécrose d'une partie du segment 6 du foie secondaire à une plaie par arme à feu chez un patient référé de la région de Diffa où sévit l’insurrection de Boko Haram.

Le transport des patients du lieu de la blessure à l'hôpital est assuré par les proches du patient (en taxi ou en véhicule personnel) dans 84,32\% des cas dans notre série bien qu'un service d'aide médicale urgente (SAMU) existe à Niamey. Le Niger à l'instar de certains pays en voie de développement se doit de planifier et de développer un 'trauma system', avec un service d'ambulance formel couvrant à terme tout le Niger (Zakariah, 2017) et offrant des soins préhospitaliers adéquats. En effet plus d'un blessé grave sur 2 meurt dans nos pays par défaut de soins préhospitaliers adéquats (Mock, 1992). En plus il faudrait aussi penser à rendre accessible le bilan morphologique en urgence ; en effet $11,70 \%$ des patients de notre série avaient eu un scanner ; de manière à pouvoir assurer un bilan lésionnel exhaustif ce qui permettra de réduire le taux de laparotomie non thérapeutique. Dans notre série les indications opératoires était l'éviscération, l’instabilité hémodynamique, la péritonite, l'issue de liquide digestif par la plaie et la laparotomie exploratrice avec $19,56 \%$ de laparotomie non thérapeutique toutes indications comprises. Toute fois toutes les plaies abdominales par arme à feu (17,65\% des patients) avaient bénéficié d'une laparotomie exploratrice. Dans notre contexte toutes les plaies abdominales par arme à feu sont encore traitées par laparotomie exploratrice même si la faisabilité d'un traitement non opératoire de blessés par arme à feu sélectionnés a été démontrée (Jansen, 2013), (Lamb, 2014). Il 
est communément admis en cas de plaie abdominale que l'instabilité hémodynamique persistante, l'éviscération, la péritonite et l'empalement constituent des indications opératoires immédiates (Biffl, 2015), (Biffl, 2010). Il convient d'associer à ces indications opératoires immédiates l'issue par la plaie du contenu d'un organe creux avant la constitution de la péritonite secondaire à la lésion sous jacente. Ce qui pourrait permettre d'explorer et de réparer les éventuelles lésions avant la généralisation de la péritonite et ses conséquences.

Les plaies de l'abdomen entrainent des lésions viscérales multiples qui concernent surtout le tractus digestif. Mais les lésions associées sont aussi fréquentes et parfois graves ; dans notre série chaque blessé avait au moins une lésion associée et cette association lésionnelle réalisait un polytraumatisme chez $21,57 \%$ de nos patients. La fréquence et la gravité de ces lésions (lésions du contenu abdominal et lésions associées) sont fonction du mécanisme lésionnel (Störmann, 2016). Les armes à feu avaient occasionné en moyenne 3 lésions par patient dans notre série et une mortalité 1,5 fois plus élevée que les autres agents vulnérants. La mortalité globale dans notre série était de 13,73\%. Les causes de décès étaient dominées par le défaut de compensation des pertes sanguines au cours de la prise en urgence. Ainsi la majorité des décès serait évitable par la mise en œuvre d'un protocole de transfusion massive.

\section{Conclusion}

A l'hôpital national de Niamey les plaies de l'abdomen sont souvent dues à une agression par arme blanche. Elles peuvent être isolées ou survenir dans un contexte de polytraumatisme. Ce blessé parfois instable est transporté à l'hôpital dans un véhicule non médicalisé sans soins préhospitaliers. La réalisation du bilan morphologique complet est aléatoire rendant difficile ''l'abstentionnisme sélectif'. La morbimortalité est élevée avec une proportion importante de décès évitable.

\section{References:}

1. P. Störmann, K. Gartner, H. Wyen, T. Lustenberger, I. Marzi, et S. Wutzler, «Epidemiology and outcome of penetrating injuries in a Western European urban region », Eur. J. Trauma Emerg. Surg., vol. 42, no 6, p. 663-669, déc. 2016.

2. R. Fanomezantsoa et al. "Traumatismes fermés et pénétrants de l'abdomen: analyse rétrospective sur 175 cas et revue de la littérature », Pan Afr. Med. J., vol. 20, nº 129, 162015.

3. C. Coquery-Vidrovitch, "De la ville en Afrique noire », Ann. Hist. Sci. Soc., vol. 61e année, $n^{0}$ 5, p. 1087-1119, 2006. 
4. B. Vincent et B. Julien, «Les "crises sahéliennes" entre perceptions locales et gestions internationales », Polit. Afr., vol. 130, n 2, p. 5-22, 2013.

5. W. L. Biffl et A. Leppaniemi, "Management Guidelines for Penetrating Abdominal Trauma », World J. Surg., vol. 39, $\mathrm{n}^{0}$ 6, p. 1373-1380, juin 2015.

6. S. Barbois et al., « Management of penetrating abdominal and thoracoabdominal wounds: A retrospective study of 186 patients. », J. Visc. Surg., vol. 153, n 4 Suppl, p. 69-78, août 2016.

7. E. J. MacKenzie et al., «A National Evaluation of the Effect of Trauma-Center Care on Mortality », N. Engl. J. Med., vol. 354, nº 4, p. 366-378, janv. 2006.

8. R. Sani R, Ngo Bissemb NM, Illo A, Souna B, Baoua BM, Bazira L, « La plaie abdominale. Revue de 316 dossiers à l'Hôpital National de Niamey - Niger ». Med Afr Noir. Vol. 51 n7, p 399-402, 2004

9. Thane Ngo Naomie MB, « Traumatismes abdominaux à propos de 676 cas à l'hôpital national de Niamey », Thèse de médecine, Université Abdou Moumouni de Niamey, Niamey, 2002.

10. Ayité A, Eteye K, Feteke L, Tchatagba K, Senah K, Attipou K, Bissang $\mathrm{K}$, James K, « Les plaies pénétrantes de l'abdomen au CHU de Lomé. A propos de 44 cas. » Med Afr Noire. Vol. 43 n¹2, p 642-646, 1996

11. A. Zakariah, B. T. Stewart, E. Boateng, C. Achena, G. Tansley, et C. Mock, "The Birth and Growth of the National Ambulance Service in Ghana », Prehospital Disaster Med., vol. 32, nº 1, p. 83-93, févr. 2017.

12. [12] C. N. Mock, G. J. Jurkovich, D. nii-Amon-Kotei, C. ArreolaRisa, et R. V. Maier, « Trauma Mortality Patterns in Three Nations at Different Economic Levels: Implications for Global Trauma System Development », J. Trauma Acute Care Surg., vol. 44, nº 5, p. 804, mai 1998.

13. J. O. Jansen et al., "Selective non-operative management of abdominal gunshot wounds: Survey of practise », Injury, vol. $44, n^{0} 5$, p. 639-644, mai 2013.

14. C. M. Lamb et J. P. Garner, « Selective non-operative management of civilian gunshot wounds to the abdomen: A systematic review of the evidence », Injury, vol. 45, no 4, p. 659-666, avr. 2014.

15. W. L. Biffl et E. E. Moore, « Management guidelines for penetrating abdominal trauma », Curr. Opin. Crit. Care, vol. 16, nº 6, p. 609, déc. 2010. 